\title{
Anxiety and depressive symptoms in women and men from early pregnancy to 30 months postpartum
}

\author{
Catarina Canário (iD $\ddagger$ and Bárbara Figueiredo (iD \\ School of Psychology, University of Minho, Braga, Portugal
}

\begin{abstract}
Aims: The aims of this study were to analyse the changes in anxiety and depression symptoms from early pregnancy to 30 months postpartum according to gender and parity.

Method: 129 couples $(N=258)$ recruited from an obstetrics unit completed self-report measures of anxiety and depression at each pregnancy trimester, childbirth, 3 and 30 months postpartum. Using multilevel modelling, piecewise dyadic growth curve models were performed, assessing time, gender and parity as predictors of anxiety and depression symptoms.

Results: Anxiety and depression symptoms decreased from the first trimester to 3 months postpartum and increased from 3 to 30 months postpartum, returning to the baseline levels in the overall sample. The symptoms were positively correlated within-dyad; in a given time point when a partner reported more symptoms, the other reported more symptoms as well. Changes in anxiety and depression symptoms over time were different according to gender and parity, especially from 3 to 30 months postpartum. Primiparous women revealed low stable symptoms, whereas multiparous women revealed the steepest symptoms increase (in comparison to primiparous men and women and multiparous men).

Conclusions: This study corroborates the literature considering that the transition to parenthood can last until the child's age of 2 or 3 years. Results point out that the risk for anxiety and depression symptoms increasing over the postpartum period is greater for multiparous and lesser for primiparous women. Future studies should explore the factors that contribute to the high risk of symptoms increase over the postpartum period for multiparous women. Screening and intervention should target couples and not only women.
\end{abstract}

\section{ARTICLE HISTORY}

Received 14 May 2016

Accepted 6 June 2017

\section{KEYWORDS}

Anxiety and depression symptoms; pregnancy and postpartum; gender and parity; dyadic growth curve analyses

\section{Introduction}

Even though the transition to parenthood is acknowledged to be a normative life transition, because it is expected and experienced by the vast majority of individuals and/or families in the population (Cowan, 1991), it also represents a challenging period, requiring individual and dyadic adjustment to several bio-psychosocial changes. Family distress and adaptation can take place across several interrelated domains, which include the self, the couple and 
the family (Cowan \& Cowan, 2012; Cowan, Cowan, Heming, \& Miller, 1991). One of these domains regards the characteristics of each parent, and includes aspects such as self-concept, self-esteem, symptoms of depression and anxiety and emotional distress (Cowan \& Cowan, 2012; Cowan et al., 1991).

In terms of the sense of self, one of the major challenges for individuals, both women and men, is to cope with the impact of psychological changes (Cowan et al., 1991). As the transition to parenthood can last until the child's age of 2-3 years (Cowan et al., 1991), it is important to study the symptoms of depression and anxiety in both men and women from pregnancy through the first three years postpartum, due to the fact that these symptoms have been found to significantly affect factors such as the couple's relationship quality, parent-child interaction and the overall child's development (e.g. Dubber, Reck, Müller, \& Gawlik, 2015; Giallo, Woolhouse, Gartland, Hiscock, \& Brown, 2015; Parfitt \& Ayers, 2009).

The adaptation to this transition is not limited to the first child - it also takes place when a second or third child becomes part of the family. Recent literature suggests that psychological difficulties might exist not only for primiparous women but also for multiparous women (e.g. Dipietro, Costigan, \& Sipsma, 2008; Sutter-Dallay, Cosnefroy, Glatigny-Dallay, Verdoux, \& Rascle, 2012). Nevertheless, the particular challenges that pregnancy and the postpartum period pose to primiparous and multiparous women and men, both individually and as a dyad, in terms of their depression and anxiety symptoms remain unclear.

Anxiety and depression are two different conditions that can either occur independently or coexist (American Psychiatric Association, 2013). The symptoms have frequently been jointly assessed in studies focused on the transition to parenthood, as they have often been found to be comorbid during this period (e.g. Heron, O'Connor, Evans, Golding, \& Glover, 2004; Kessler, Keller, \& Wittchen, 2001; Wenzel, 2011).

Despite the great number of studies addressing anxiety and depression symptoms over pregnancy and the postpartum period, conflicting evidence exists regarding the course of symptoms over these periods. Some studies indicated an increase in women's and men's depression symptoms from pregnancy to 12 months postpartum (e.g. Matthey, Barnett, Ungerer, \& Waters, 2000), and in men's depression symptoms from pregnancy to 6 months postpartum (e.g. Paulson \& Bazemore, 2010). Other studies revealed the stability of women's anxiety and depression symptoms over pregnancy and the postpartum period (e.g. Giardinelli et al., 2012; Heron et al., 2004). Recent studies identified a decrease in women's anxiety symptoms across pregnancy (e.g. Buist, Gotman, \& Yonkers, 2011; Matthey \& Ross-Hamid, 2012), and a decrease in women's anxiety and depression symptoms from pregnancy to 24 months postpartum (Dipietro et al., 2008). Further studies reported a decrease in both women's and men's anxiety and depression symptoms from pregnancy to 6 months postpartum (Whisman, Davila, \& Goodman, 2011).

Women's anxiety and/or depression symptoms during pregnancy and the postpartum period have been studied extensively. Only recently, research has also begun to look at men and determined that $10 \%$ of new fathers experience mental health difficulties, in addition to reporting that paternal postnatal depression during the first year postpartum is predicted by parenting distress, marital relationship quality and parenting efficacy (e.g. deMontigny, Girard, Lacharité, Dubeau, \& Devault, 2013; Singley \& Edwards, 2015). Furthermore, only recently has research started to include both members of the couple as participants, and point out that women's and men's postpartum depression symptoms are positively 
associated (e.g. Don \& Mickelson, 2012; Kerstis, Engström, Edlund, \& Aarts, 2013; Perren, von Wyl, Bürgin, Simoni, \& von Klitzing, 2005).

Women were usually found to exhibit higher anxiety and depression symptoms than men at the third pregnancy trimester, childbirth, 3 and 12 months postpartum (Escriba-Aguir \& Artazcoz, 2011; Escribe-Aguir, Gonzalez-Galarzo, Barona-Vilar, \& Artazcoz, 2008); however, data are somewhat inconsistent regarding gender effects over time. Some studies did not identify gender effects on anxiety and depression symptoms over pregnancy and/or the postpartum period (e.g. Parfitt, Pike, \& Ayers, 2013; Perren et al., 2005), while in other studies, anxiety symptoms were found to remain stable for women while decreasing for men from late pregnancy to 12 months postpartum (Keeton, Perry-Jenkins, \& Sayer, 2008). Furthermore, in other studies depression symptoms were found to increase until 12 (Keeton et al., 2008) or to decrease until 24 months postpartum (Rholes et al., 2011) in women while remaining stable for men from late pregnancy to 12 and 24 months postpartum.

Studies assessing parity differences on anxiety and depression symptoms over pregnancy and the postpartum period are rare and include mostly women (e.g. Di Florio et al., 2014; Melo et al., 2012; Sutter-Dallay et al., 2012). As far as we can ascertain, data on the differences of parity on men's symptoms over pregnancy and the postpartum period are limited to a single study determining lower psychological symptoms in primiparous men when compared to multiparous men at the third trimester of pregnancy (Condon \& Esuvaranathan, 1990). Moreover, results regarding parity effects on anxiety and depression symptoms over pregnancy and the postpartum period are controversial. While some studies found no differences in depression symptoms between primiparous and multiparous women during the puerperium (e.g. Breitkopf et al., 2006; Giardinelli et al., 2012), others reported more anxiety (Zanardo, Gasparetto, Giustardi, \& Suppiej, 2009) and depression (Di Florio et al., 2014) symptoms in primiparous women during the puerperium, whereas further studies revealed more depression symptoms in multiparous women during pregnancy (Melo et al., 2012; Sutter-Dallay et al., 2012). Regarding the symptom trajectories, from 6 weeks to 24 months postpartum, Dipietro et al. (2008) found an increase in primiparous women's anxiety symptoms, and a decrease in multiparous women's anxiety and depression symptoms.

In a couple, both members of the dyad mutually influence each other: one partner affects and is affected by the other partner (Kenny, Kashy, \& Cook, 2006). The interdependence between partners refers to this causal influence happening to the members of a couple and has recently been identified for anxiety and/or depression symptoms experienced in situations other than the transition to parenthood (e.g. Chung, Moser, Lennie, \& Rayens, 2009; Dekel et al., 2014; Parker, Johnson, \& Ketring, 2012). Despite some studies identifying positive correlations between men's and women's anxiety and/or depression symptoms over pregnancy and/or the postpartum period, interdependence between partners was usually not considered (e.g. Areias, Kumar, Barros, \& Figueiredo, 1996; Dudley, Roy, Kelk, \& Bernard, 2001; Keeton et al., 2008). To the best of our knowledge, to date, only two studies have contemplated the interdependence between partners for anxiety and depressive symptoms during pregnancy and the postpartum period. Anxiety symptoms were found to be positively correlated within-dyad, as opposed to depression symptoms that were found to be negatively correlated within-dyad (Don, Chong, Biehle, \& Mickelson, 2014; Rholes et al., 2011).

Given that few studies focused on anxiety or depression symptoms over the transition to parenthood included several assessment waves over pregnancy and the postpartum period, and that fewer studies considered gender, parity and within-dyad interdependence, 
the purpose of the present study was to analyse the individual and dyadic trajectories of men's and women's anxiety and depression symptoms, using dyadic growth curve models: (a) from the first trimester to 30 months postpartum, (b) assessing gender and parity differences and effects, and (c) examining the interdependence between partners' symptoms.

\section{Method}

\section{Participants}

This study followed a sample of couples surveyed from the first trimester until 30 months postpartum. Three hundred and fifty-seven pregnant women were invited to integrate the study in an Obstetrics Unit at their first pregnancy trimester appointment. Even though 300 women agreed to participate, only a sample of 260 couples was recruited, corresponding to the situations where both the pregnant women and her partner agreed to participate. Most of these participants were Portuguese (91.4\%), aged between 20 and 39 years old (85.9\%), from low or medium-low socioeconomic levels (57.4\%), had nine or more years of education (67.2\%), were employed (80.4\%), married or cohabiting $(88.4 \%)$ and primiparous (55\%), as presented in Table 1.

The study had six assessment waves, at the first (between weeks 8 and 14), second (between weeks 20 and 24) and third ((between weeks 30 and 34) pregnancy trimester, childbirth (between days 1 and 3), 3 (between weeks 10 and 14) and 30 months (between months 24 and 36). A total of 215 couples ( $n=430,82.7 \%)$ completed all assessment waves until 3 months postpartum, and a total of 129 couples completed all assessment waves until 30 months postpartum ( $n=258,49.6 \%$ ). Participants who completed all assessments were

Table 1. Participants' sociodemographic characterization at baseline.

\begin{tabular}{|c|c|c|c|c|c|}
\hline & Men & Women & Primiparous & Multiparous & Total \\
\hline & $n=260 \%$ & $n=260 \%$ & $n=286 \%$ & $n=234 \%$ & $n=520 \%$ \\
\hline \multicolumn{6}{|l|}{ Age (years) } \\
\hline$\leq 19$ & 4.2 & 11.5 & 12.4 & 2.6 & 7.9 \\
\hline $20-29$ & 38.9 & 47.0 & 51.6 & 33.3 & 42.9 \\
\hline $30-39$ & 46.9 & 39.2 & 33.9 & 55.4 & 43.1 \\
\hline$\geq 40$ & 10.0 & 2.3 & 2.1 & 8.7 & 6.1 \\
\hline \multicolumn{6}{|l|}{ Marital status } \\
\hline Cohabiting & 32.5 & 32.6 & 35.8 & 28.5 & 32.5 \\
\hline Married & 55.7 & 55.8 & 49.7 & 63.2 & 55.8 \\
\hline Single & 10.2 & 11.2 & 14.5 & 6.1 & 10.7 \\
\hline Divorced & 1.6 & .4 & 0 & 2.2 & 1.0 \\
\hline \multicolumn{6}{|l|}{ Socioeconomic level } \\
\hline High & 9.3 & 16.4 & 15.6 & 8.9 & 12.5 \\
\hline $\begin{array}{l}\text { Medium- } \\
\text { high }\end{array}$ & 14.0 & 7.4 & 9.4 & 13.0 & 11.1 \\
\hline Medium & 18.6 & 19.6 & 16.5 & 21.9 & 19.0 \\
\hline Medium-low & 30.3 & 26.4 & 30.4 & 26.5 & 28.6 \\
\hline Low & 27.8 & 30.2 & 28.1 & 27.7 & 28.8 \\
\hline \multicolumn{6}{|l|}{ Occupational status } \\
\hline Employed & 89.0 & 71.8 & 77.7 & 83.5 & 80.4 \\
\hline Unemployed & 9.0 & 21.6 & 20.0 & 13.4 & 15.4 \\
\hline Household/student & 2.0 & 6.6 & 5.3 & 3.1 & 4.2 \\
\hline \multicolumn{6}{|l|}{ Years of education } \\
\hline$<9$ & 38.6 & 26.9 & 29.3 & 37.8 & 32.8 \\
\hline $9-12$ & 47.9 & 54.3 & 53.0 & 50.0 & 51.0 \\
\hline$>12$ & 13.5 & 18.8 & 17.7 & 12.2 & 16.2 \\
\hline
\end{tabular}


more likely to be older, $X^{2}(3)=33.05, p<.001$, employed, $X^{2}(6)=16.32, p=.012$, belong to medium or high socioeconomic levels, $X^{2}(2)=26.45, p<.001$, and have more years of education, $X^{2}(2)=29.02, p<.001$. Also, they reported fewer anxiety and depression symptoms at the first trimester, $t(503)=2.19, p=.03, t(503)=4.05, p<.001$, but not at 3 months postpartum.

Most of the mothers delivered their babies after the 37th week of gestation (97\%), began to breastfeed at childbirth (95.7\%) and reported good health and developmental outcomes of their child at 30 months postpartum (95\%).

\section{Measures}

\section{Sociodemographic questionnaire}

Information about the participants (e.g. age, matrimonial status, socioeconomic level, occupational status and years of education) was collected using a sociodemographic questionnaire (Figueiredo, Teixeira, Conde, Pinto, \& Sarmento, 2009).

\section{State-trait anxiety inventory}

The State-Trait Anxiety Inventory (STAl; Spielberger, Gorsuch, Lushene, Vagg, \& Jacobs, 1983) is a self-report questionnaire that consists of two subscales, the state anxiety and the trait anxiety, each including 20 items (scored on a four-point Likert scale from 1 to 4). The state anxiety subscale (STAI-S), designed for measuring the temporary condition of anxiety (in a specific situation) was used in this study. Scores equal to or higher than 42 are considered to screen for anxiety states, focusing merely on the reported symptoms (Biaggio, Natalicio, \& Spielberger, 1976) in women and men. A recent study with a Portuguese sample demonstrated reasonable validity of the STAI as a screening tool for anxiety in women over pregnancy and postpartum, pointing out an optimal cut-off value of 40 for pregnancy and of 34 for the postpartum period (Tendais, Costa, Conde, \& Figueiredo, 2014). The STAI Portuguese version showed good internal consistency ( $\alpha$ ranged from .87 to .93) (Biaggio et al., 1976). In the present sample, coefficient alphas ranged from .88 to .94 for men and women.

\section{Edinburgh Postnatal Depression Scale}

The Edinburgh Postnatal Depression Scale (EPDS; Cox, Holden, \& Sagovsky,1987) is a selfreport questionnaire composed of 10 items (scored on a four-point Likert scale from 0 to 3 ) to assess the existence and severity of depression symptoms. The scores range between 0 and 30. Scores equal or higher than 10 are considered to indicate depression (Cox et al., 1987). A recent study with a Portuguese sample demonstrated reasonable validity of the EPDS as a screening tool for depression in women over pregnancy and postpartum, pointing out an optimal cut-off value of 9 for pregnancy and of 7 for the postpartum period (Tendais et al., 2014). The EPDS Portuguese version showed good internal consistency (Cronbach's alpha of .85) (Figueiredo, Pacheco, \& Costa, 2007). The coefficient alphas in the present sample ranged from .78 to .88 for men and women.

\section{Procedure}

This research received previous approval from the institution Ethical Commission. Participants were recruited in an Obstetrics outpatients unit at their first pregnancy appointment. The exclusion criteria were not reading or writing Portuguese and multiple gestations. The aims 
and the procedures of the study were explained. After signing an informed consent, the sociodemographic questionnaire, STAI and EPDS were separately administered to the pregnant woman and partner (between weeks 8 and 14, $M=13.90, \mathrm{SD}=1.38$ ). The STAI and the EPDS were then sent by letter or email, using an electronic form, during the second (between weeks 20 and $24, M=21.21, S D=1.14$ ) and third pregnancy trimester (between weeks 30 and $34, M=31.14, S D=1.65$ ), as well as at childbirth (between days 1 and $3, M=2.30$, $\mathrm{SD}=0.51$ ), at 3 (between weeks 10 and $14, M=13.60, \mathrm{SD}=0.80$ ) and 30 months (between weeks 24 and $36, M=30.20$, SD $=5.51$ ) postpartum.

\section{Data structure and analytic model}

Statistical analysis was performed with IBM SPSS Statistics for Windows, v.19.0. Descriptive statistics were performed for each outcome variable at each assessment wave (Table 2). Bivariate Pearson correlations were performed to assess correlations between men and women at each assessment wave, within-men correlations and within-women correlations (Table 3). Paired-sample $t$-tests were performed to compare the outcome variables between the first and last assessment waves.

Dyadic growth curve models were tested using multilevel modelling (MLM; Kenny et al., 2006) to estimate women's and men's trajectories of change for anxiety and depression symptoms over time, and symptoms' within-dyad interdependence. Two-level models ${ }^{1}$ were estimated for anxiety and depression symptoms (outcome variables), in which level 1 referred to the participants' score at each time point and level 2 to the dyad. Individual differences for men and women were modelled at the dyad level (Kenny et al., 2006).

In each model, time 0 was defined as the date of the first assessment at the first trimester (baseline) and the time variable was scored in months since the baseline until the child's age of 30 months. Piecewise linear growth curve models (Duncan, Duncan, \& Strycker, 2006) were performed to assess the changes on the outcome variables at baseline, from baseline

Table 2. Means for anxiety and depressive symptoms for men and women, primiparous and multiparous.

\begin{tabular}{|c|c|c|c|c|c|c|c|c|}
\hline \multirow[b]{2}{*}{ Symptoms } & \multicolumn{2}{|c|}{ Men } & \multicolumn{2}{|c|}{ Women } & \multicolumn{2}{|c|}{ Primiparous } & \multicolumn{2}{|c|}{ Multiparous } \\
\hline & $M$ & $S D$ & $M$ & $S D$ & $M$ & $S D$ & $M$ & $S D$ \\
\hline \multicolumn{9}{|l|}{ Anxiety } \\
\hline 1st trimester & 33.10 & 8.83 & 36.04 & 8.88 & 33.91 & 8.92 & 35.39 & 8.87 \\
\hline 2nd trimester & 31.17 & 8.07 & 34.68 & 9.37 & 31.51 & 7.90 & 34.76 & 9.79 \\
\hline 3rd trimester & 32.61 & 7.95 & 36.24 & 9.03 & 32.90 & 7.98 & 36.46 & 9.17 \\
\hline Childbirth & 32.72 & 9.13 & 35.76 & 10.25 & 33.99 & 10.61 & 34.62 & 8.77 \\
\hline $\begin{array}{l}3 \text { months } \\
\text { postpartum }\end{array}$ & 29.37 & 7.99 & 30.84 & 8.36 & 28.99 & 7.80 & 31.51 & 8.49 \\
\hline $\begin{array}{l}30 \text { months } \\
\text { postpar- } \\
\text { tum }\end{array}$ & 33.77 & 9.43 & 34.45 & 9.48 & 32.31 & 8.28 & 36.79 & 10.41 \\
\hline \multicolumn{9}{|l|}{ Depressive } \\
\hline 1st trimester & 5.24 & 3.96 & 6.42 & 4.19 & 5.42 & 4.08 & 6.34 & 4.07 \\
\hline 2nd trimester & 4.18 & 3.31 & 6.00 & 4.32 & 4.74 & 3.86 & 5.59 & 4.07 \\
\hline 3rd trimester & 4.08 & 3.26 & 5.49 & 4.25 & 4.30 & 3.75 & 5.45 & 3.92 \\
\hline Childbirth & 4.06 & 3.73 & 5.87 & 4.67 & 4.72 & 4.39 & 5.30 & 4.23 \\
\hline $\begin{array}{l}3 \text { months } \\
\text { postpar- } \\
\text { tum }\end{array}$ & 3.51 & 3.46 & 4.72 & 4.21 & 3.62 & 3.73 & 4.75 & 4.03 \\
\hline $\begin{array}{l}30 \text { months } \\
\text { postpar- } \\
\text { tum }\end{array}$ & 4.80 & 4.21 & 6.11 & 4.36 & 4.82 & 3.84 & 6.47 & 4.82 \\
\hline
\end{tabular}


Table 3. Correlations among anxiety and depressive symptoms for men and women.

\begin{tabular}{|c|c|c|c|c|c|c|}
\hline & 1st trimester & 2nd trimester & 3rd trimester & Childbirth & $\begin{array}{c}3 \text { months } \\
\text { postpartum }\end{array}$ & $\begin{array}{l}30 \text { months } \\
\text { postpartum }\end{array}$ \\
\hline \multicolumn{7}{|l|}{ Anxiety symptoms } \\
\hline 1st trimester & .16 & .54 & .57 & .51 & .43 & .34 \\
\hline 2nd trimester & .60 & .31 & .65 & .44 & .54 & .34 \\
\hline 3rd trimester & .54 & .59 & .41 & .59 & .55 & .39 \\
\hline Childbirth & .36 & .36 & .52 & .52 & .56 & .25 \\
\hline 3 months postpartum & .41 & .39 & .51 & .49 & .31 & .38 \\
\hline 30 months postpartum & .36 & .31 & .47 & .28 & .40 & .49 \\
\hline \multicolumn{7}{|l|}{ Depressive symptoms } \\
\hline 1st trimester & .31 & .69 & .67 & .60 & .51 & .37 \\
\hline 2nd trimester & .62 & .32 & .69 & .60 & .54 & .37 \\
\hline 3rd trimester & .49 & .70 & .37 & .68 & .54 & .50 \\
\hline Childbirth & .49 & .59 & .65 & .55 & .60 & .50 \\
\hline 3 months postpartum & .46 & .62 & .63 & .69 & .44 & .46 \\
\hline 30 months postpartum & .45 & .51 & .56 & .53 & .51 & .42 \\
\hline
\end{tabular}

to 3 months postpartum, and from 3 to 30 months postpartum. Two different multilevel models were performed to predict anxiety and depression symptoms. Each model included time from the first trimester to 3 months postpartum, time from 3 to 30 months postpartum, gender and parity, and all possible interactions, as predictors of the outcome variables (Table 4, Figures 1 and 2). The fixed and random effects are presented in Table 4. The deviance difference tests revealed that the models provided good fits to the data. Results were reported at a $p<.05$ significance level.

\section{Results}

\section{Preliminary analyses}

Descriptive statistics (mean and standard deviation) for men's and women's, primiparous and multiparous parents' anxiety and depression symptoms at each assessment wave are presented in Table 2.

Correlations among partners' symptoms for all assessment waves were statistically significant, ps <.001 (Table 3). Men's and women's scores for the outcome variable at each assessment wave were positively correlated. Regarding anxiety and depression symptoms, between partner's correlations at each time point were medium to high size $\left(r_{\text {range }}=.31-.55\right)$, except for the correlation between women's and men's anxiety symptoms at the first trimester, which was small $(r=.16)$. Additionally, within-men and within-women correlations of the outcome variables show that men's and women's anxiety and depression symptoms are related over time. Within-women and within-men correlations of anxiety symptoms ranged from small $\left(r_{\text {women }}=.28 ; r_{\text {men }}=.25\right)$ to high size $\left(r_{\text {women }}=.60 ; r_{\text {men }}=.65\right)$. Within-women and within-men correlations of depression symptoms ranged from medium $(r$ women $=.45$; $\left.r_{\text {men }}=.37\right)$ to high size $\left(r_{\text {women }}=.70 ; r_{\text {men }}=.69\right)$. 


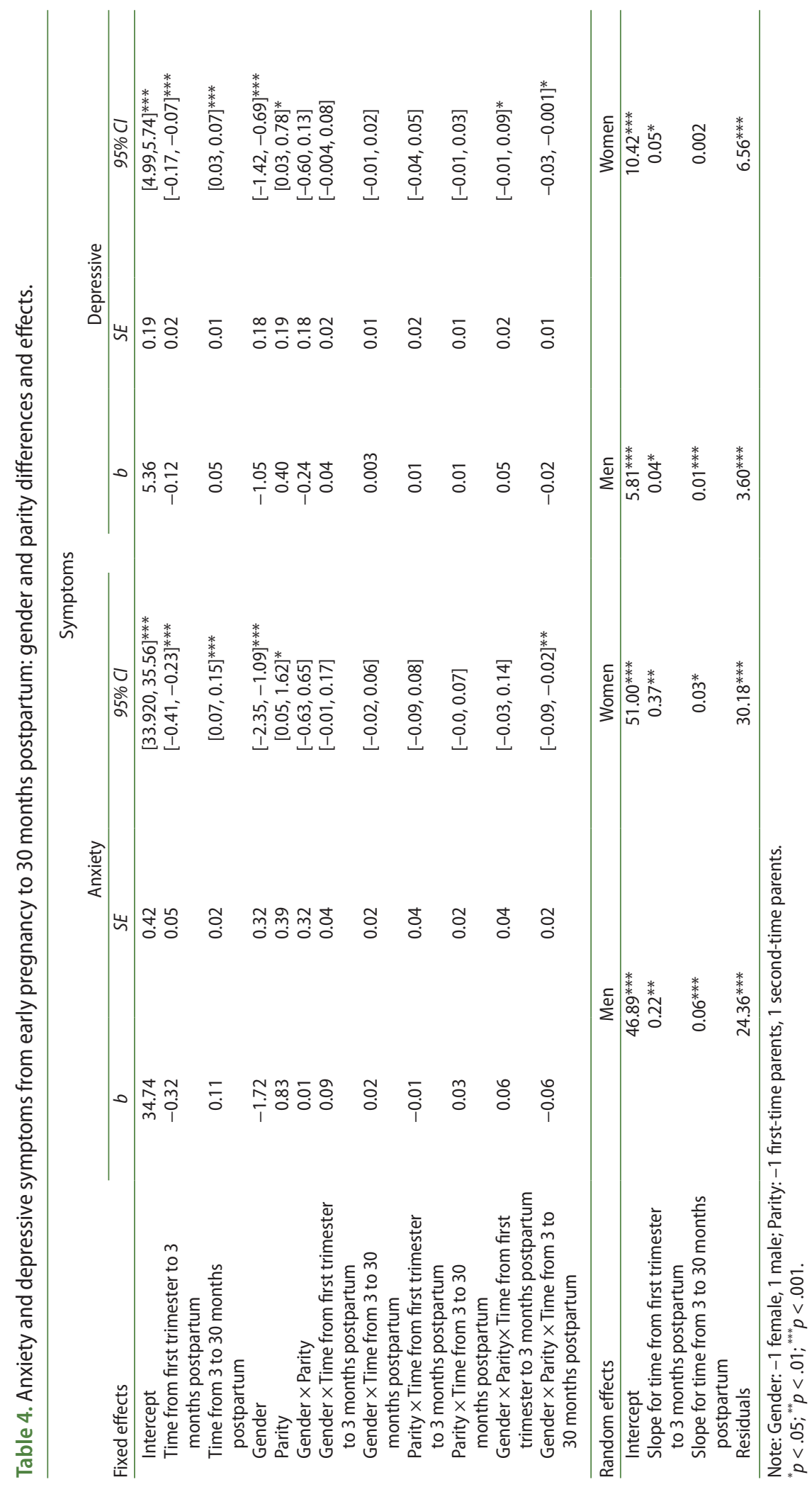




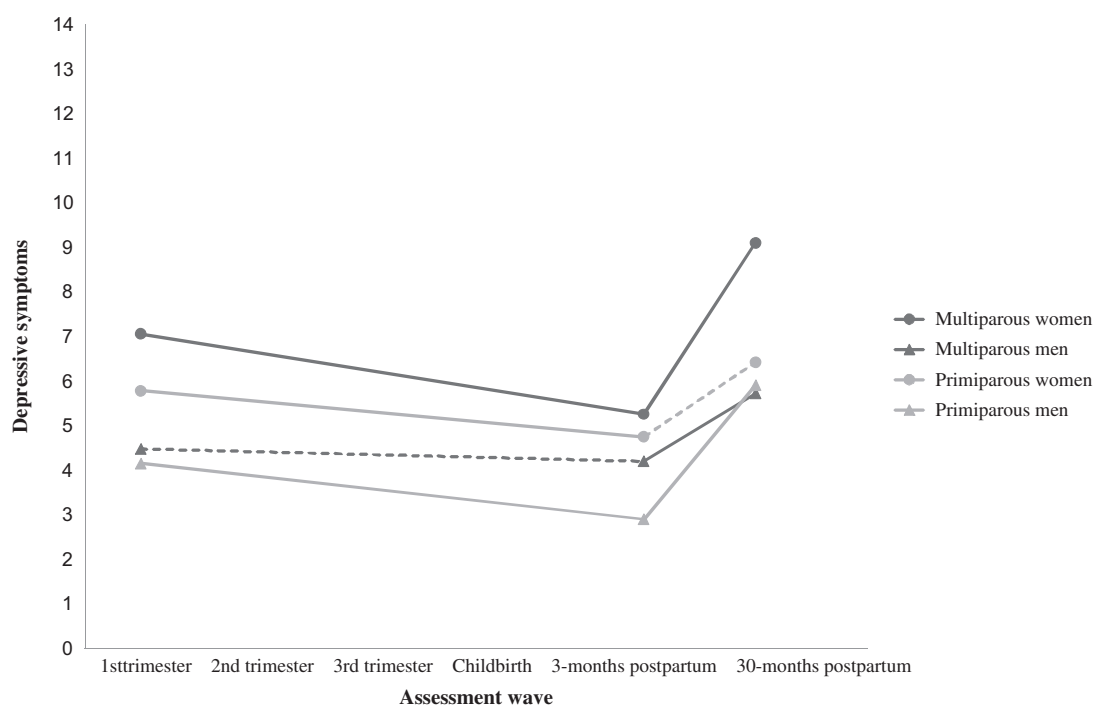

Figure 1. Depressive symptoms from early pregnancy to postpartum: gender and parity differences and effects. Significant differences are presented with a continuous line.

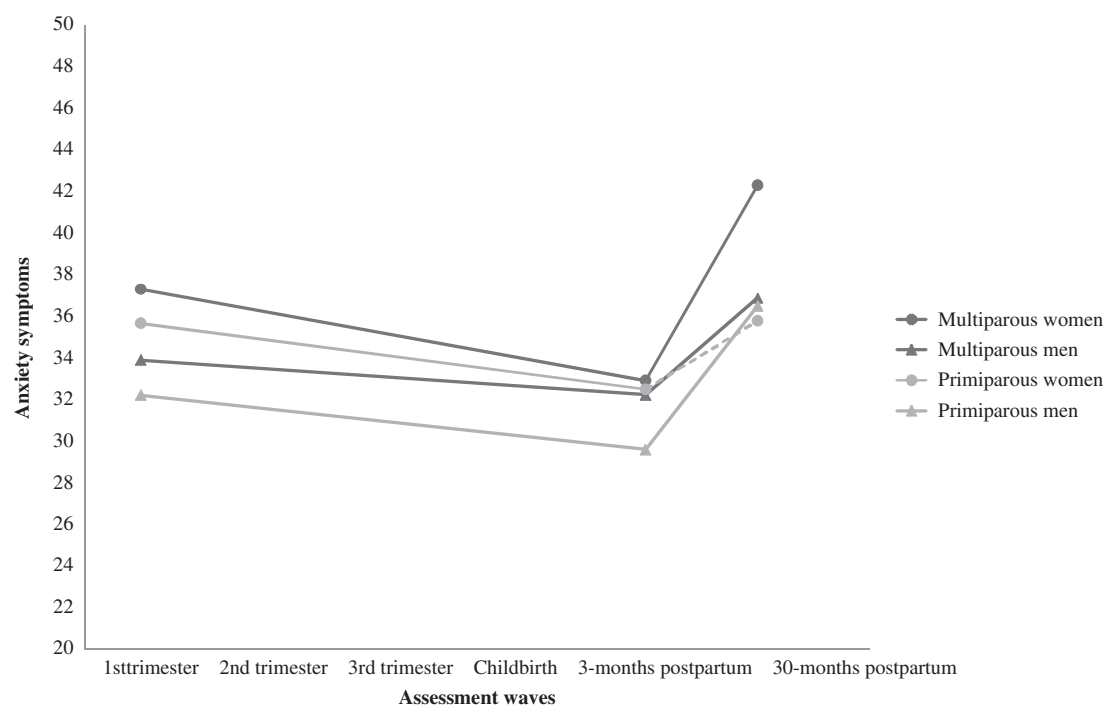

Figure 2. Anxiety symptoms from early pregnancy to postpartum: gender and parity differences and effects. Significant differences are presented with a continuous line.

\section{Anxiety and depression symptoms from early pregnancy to 30 months postpartum}

Main effects of time were found in both anxiety and depression symptom models (Table 4). From the first trimester to 3 months postpartum, anxiety and depression symptoms decreased (the average person's anxiety and depression symptoms decreased 0.32 units and 0.12 units per month, respectively). From 3 to 30 months postpartum, anxiety and depression symptoms increased (the average person's anxiety and depression symptoms 
increased 0.11 units and 0.05 units per month). However, anxiety, $t(243)=-0.62, p=.53$ and depression, $t(243)=-1.44, p=.15$ symptoms were not different between the first and the last assessment waves.

\section{Anxiety and depression symptoms from early pregnancy to 30 months postpartum: gender and parity differences and effects}

The main effects for gender were found in both anxiety and depression symptom models (Table 4). At the first trimester, women had more anxiety (mean difference of 3.43) and depression (mean difference of 2.10) symptoms than did men. No other gender effects were found for time regarding both symptoms.

The main effects of parity were also found in both models (Table 4). At baseline, multiparous parents had more anxiety (mean difference of 1.63) and depression (mean difference of 0.80 ) symptoms than did primiparous parents. As presented in Table 4, a significant interaction among time from the first trimester to 3 months postpartum, gender and parity was found in the depression model, and one significant interaction among time from 3 to 30 months postpartum, gender and parity, was found in both models.

As depicted in Figure 1, depression symptoms decreased from the first trimester to 3 months postpartum for primiparous men and women, and for multiparous women, but not for multiparous men. Specifically, primiparous men and women and multiparous women depression symptoms decreased $0.20, p<.001,0.11, p=.006$ and $0.14, p<.001$, units per month. Pairwise comparisons revealed that, compared to the other groups' symptoms, multiparous women's had a steeper depression, $p=.04$, symptoms decrease from the first trimester to 3 months postpartum.

Both anxiety (Figure 2) and depression (Figure 1) symptoms increased from 3 to 30 months postpartum for multiparous men and women, and for primiparous men, but not for primiparous women. Specifically, multiparous men's anxiety and depression symptoms increased 0.11 units per month, $p=.02$, and 0.05 units per month, $p=.03$, respectively; multiparous women's anxiety and depression symptoms increased 0.18 units per month, $p<.001$, and 0.07 units per month, $p<.001$, respectively; and primiparous men's anxiety and depression symptoms increased 0.15 units per month, $p<.001$ and 0.06 units per month, $p<.001$, respectively.

Pairwise comparisons revealed that, compared to the other groups' symptoms, multiparous women's presented a steeper anxiety, $p=.04$, and depression, $p=.04$, symptoms increase from 3 to 30 months postpartum.

\section{Anxiety and depression symptoms within-dyad interdependence}

The estimated residual correlation was significant for anxiety, $r=.25, Z=6.58, p<.001$, and depression, $r=.24, Z=4.19, p<.001$ symptoms, indicating that after controlling for the effects of time, gender, parity and all the covariates included in the models, if one partner reported more symptoms in a given time point, the other tended to report similar levels of symptoms.

\section{Discussion}

For the overall sample, both anxiety and depression symptoms were found to decrease from the first trimester to 3 months postpartum and to increase from 3 to 30 months postpartum. 
Symptoms were not different between the first trimester and 30 months postpartum, suggesting that despite the symptoms changing over time, they tend to return to their baseline levels. Differences in symptoms trajectories were found according to participants' gender and parity status. Whereas primiparous women revealed low stable symptoms over the postpartum period, multiparous women exhibited the steepest symptoms increase over the same period (in comparison to primiparous men and women and multiparous men), pointing out a greater probability for psychological difficulties, such as anxiety and/or depression disorders.

The results regarding the symptoms decline from the first trimester to 3 months postpartum are consistent with previous findings (e.g. Buist et al., 2011; Whisman et al., 2011). However, these are inconsistent with previous studies determining that symptoms fluctuate throughout the course of pregnancy, being higher at the final trimester (Huizink, Mulder, Robles de Medina, Visser, \& Buitelaar, 2004; Lee, Lam, Sze Mun Lau, Chong, \& Chui, 2007), and with studies identifying the stability of symptoms from pregnancy to the postpartum period (e.g. Giardinelli et al., 2012; Heron et al., 2004). This discrepancy may be explained by the methodological differences existing between studies. While the latter studies included primiparous women, comprised less than four assessment waves and, generally, used non-parametric data analytic plans, the present study comprised six assessment waves and analysed data with piecewise growth curve models, representing a robust method analysis for modelling change over time, allowing to fit a function to a set of observations (Kenny et al., 2006).

The symptoms decline from early pregnancy to 3 months postpartum may be explained by the distress experienced by women at the beginning of pregnancy, which can be transient, given the reduction of pregnancy's physical symptoms, the comforting results of routine tests and/or the diminishing fear of miscarriage, among other factors (e.g. Matthey \& RossHamid, 2012). Additionally, worries, representing an important cognitive component of anxiety, are known to decrease across pregnancy and from pregnancy to the postpartum period (e.g. Buist et al., 2011; Kishi et al., 2011), although significant worries have been reported at the end of the pregnancy related to labour and birth (Henderson \& Redshaw, 2016; Kjærgaard, Wijma, Dykes, \& Alehagen, 2008). After childbirth and until 3 months postpartum, the symptom decline may be explained by the increase in the sense of personal control and positive parent-infant interactions, which are known to lessen anxiety and depression symptoms (Keeton et al., 2008; Parfitt et al., 2013). Particularly in terms of women, the decrease in anxiety and depression symptoms may also be explained by the breastfeeding experience after childbirth. Not only has the maintenance of breastfeeding been found to be associated with a decrease in depression symptoms (Figueiredo, Canário, \& Field, 2014), but also the neuroendocrine changes inherent to breastfeeding have been related to symptoms experienced during the postpartum period. Higher blood serotonin concentrations in lactating women have been linked with the regulation of anxiety (Sekiyama, Nakatani, Yu, Seki, \& Sato-Suzuki, 2013), and have been suggested by the authors to take place through a decrease in estradiol levels after delivery and by lactation-induced brain oxytocin release during the postpartum period. Additionally, another study reported that lactating women with higher oxytocin release reveal lower anxiety and depression symptoms at 8 weeks postpartum (Stuebe, Grewen, \& Meltzer-Brody, 2013). This is particularly relevant to understand the present study's findings, as, in the Portuguese context, $98 \%$ of women initiate exclusive breastfeeding immediately after childbirth (Direção-Geral da Saúde, 2013). 
The findings related with an increase in anxiety and depression symptoms from 3 to 30 months postpartum fail to validate previous study findings (e.g. van Bussel, Spitz, \& Demyttenaere, 2009; Dipietro et al., 2008). However, they confirm and extend past findings attesting an increase in women's and men's anxiety and depression symptoms over the first postpartum year (e.g. Matthey et al., 2000; Paulson \& Bazemore, 2010), particularly after 3 months postpartum. This period seems to be more demanding for parents, as they have to address the child's needs and difficulties, and tend to experience a deterioration in the couple relationship, while facing increased environmental stressors, such as the return to work after the maternal/paternal leave of absence (e.g. Breitkopf et al., 2006; Perren et al., 2005; Whisman et al., 2011). Such strains may contribute to a symptoms' increase after 3 months postpartum, leading to a return to the baseline symptoms' levels.

Consistent with prior research is the fact that women presented more anxiety and depression symptoms at the first trimester (e.g. Escriba-Aguir \& Artazcoz, 2011; Escribe-Aguir et al., 2008). The lack of gender effects over time reinforces results from previous studies reporting no gender effects in anxiety and/or depression symptoms trajectories over pregnancy and/ or the postpartum period (e.g. Parfitt et al., 2013; Perren et al., 2005). However, the findings are inconsistent with previous studies suggesting anxiety symptoms to remain stable for women and decrease for men from late pregnancy to 12 months postpartum, whereas depression symptoms increase until 12 (Keeton et al., 2008) or decrease until 24 months postpartum (Rholes et al., 2011) in women, while remaining stable for men.

Multiparous parents presented more anxiety and depression symptoms than primiparous parents at the first pregnancy trimester, also confirmed by previous studies (e.g. Condon \& Esuvaranathan, 1990; Giardinelli et al., 2012; Melo et al., 2012). From the first trimester to 3 months postpartum, similar trajectories of anxiety symptom were found for primiparous and multiparous men's and women's symptoms (but not for primiparous women's depression symptoms). Results regarding the decrease in anxiety symptoms validate previous findings, and are consistent with other studies determining parity effects over the adaptation to pregnancy and the first postpartum weeks (e.g. Melo et al., 2012). Particularly after childbirth, this may be prompted by the positive experiences of both caregiving and interaction with the baby, which are common to all parents (Parfitt et al., 2013).

From 3 to 30 months postpartum, increases in anxiety and depression symptoms were found for multiparous men and women and primiparous men, whereas primiparous women's symptoms remained unaltered. Primiparous men revealed an increase in symptoms confirming past studies identifying an increase in depression symptoms (Garfield et al., 2014), and that $10 \%$ of new fathers exhibit mental health difficulties that include depression and anxiety disorders (Singley \& Edwards, 2015). Multiparous women presented the steepest increase in symptoms over the postpartum period. These results are consistent with previous studies pointing out parity differences to occur after 3 months postpartum, particularly in terms of higher psychological distress registered in multiparous rather than primiparous women (e.g. Sutter-Dallay et al., 2012). However, these results do not substantiate the lack of differences in maternal anxiety, stress and depression found between primiparous and multiparous women at 24 months postpartum as reported by Dipietro et al. (2008). The increase found for multiparous women reveals a greater risk for psychological difficulties, such as anxiety and/or depression disorders, experienced by this particular group. Having another child requires adaptation, not only at an individual but also couple and familiar levels (Cowan et al., 1991, p. 1). As such, multiparous women can be under more stressful 
conditions, such as the inclusion of the new baby within the family dynamics, the difficulties of adapting to more demanding processes of caregiving, the need to managing the household labour and work schedules, particularly after the maternity leave of absence, which may increase the risk for psychological difficulties (Hakulinen, Laippala, \& Paunonen, 1998). Moreover, the risk for anxiety and depression disorders has been explained by interpersonal risk factors such as lower levels of social support and marital satisfaction known to be more frequent in multiparous women (Sockol \& Battle, 2015).

Anxiety and depression symptoms were found to be positively correlated within the dyad: if one partner presented more symptoms, the other partner tended to present more symptoms as well. These results reveal the partners' interdependence regarding anxiety and depression symptoms during pregnancy and the postpartum period and determine that one's psychological adjustment affects and is affected by their partner's psychological adjustment. The results related with the interdependence of depression symptoms differ from the findings of Rholes et al. (2011), which revealed a negative correlation within-dyad, whereas the results focused on the interdependence of anxiety symptoms stand in line with the findings of Don et al. (2014). In the context of this study, symptoms' positive interdependence may occur due to the processes of multiple influence, as both partners directly affect one another, and to common fate, as both member of the dyad are exposed to the same causal factor (Kenny et al., 2006), which in this case is the birth of a child. Furthermore, the symptoms' interdependence may indirectly take place through partner relationship quality, meaning that one partner's symptoms affect the partner relationship, thus impacting upon the other partner's symptoms, and vice versa (Don \& Mickelson, 2012; Wynter, Rowe, \& Fisher, 2014)

The strengths of this study include a six-wave longitudinal design and a large sample size, in addition to comprising both primiparous and multiparous men and women, and using piecewise dyadic growth curve analysis through multilevel modelling. This study's findings have important implications for practice, namely in terms of screening and intervention. Several studies have pointed out the need to screen for anxiety and/or depression symptoms in women during pregnancy, puerperium and the early postpartum period (e.g. Farr, Dietz, O'Hara, Burley, \& Ko, 2014; Matthey, Valenti, Souter, \& Ross-Hamid, 2013; Skouteris, Wertheim, Rallis, Milgrom, \& Paxton, 2009). In light of our results, health care professionals should screen for anxiety symptoms in both men and women, especially during the postpartum period, until the child's age of 2-3 years. This is particularly relevant, because significant increase in symptoms for primiparous and multiparous men has been found to occur, as well as for multiparous women. Equally, the data supporting partners' symptom interdependence reveal that partners' symptoms change in tandem, meaning that a partner with more symptoms tends to have a partner with more symptoms as well.

The present study's findings along with previous findings reported in the literature allow us to point out a set of suggestions within the scope of the interventions targeting anxiety and depression symptoms over pregnancy and the postpartum period. Health care professionals should pay particular attention to multiparous women during the postpartum period, because this group presented a higher risk for psychological difficulties. When high-state anxiety and/ or high depression symptoms are identified, a referral should be made, and, due to the of symptoms interdependence, professionals should consider referring to couple-based interventions. Furthermore, prevention and intervention programmes aimed at reducing anxiety and depression symptoms over the transition to parenthood would benefit from including both 
primiparous and multiparous women and men, and paying particular attention to the period after 3 months postpartum. To be effective, interventions during the transition to parenthood should be developed since pregnancy, have at least five sessions, and be delivered by a highly qualified interventionist (Pinquart \& Teubert, 2010). Equally, they should include both members of the couple and provide them with information on the expected changes to their self, their relationship and family system, and also related with the difficulties and challenges posed by the transition according to gender and parity (Canário \& Figueiredo, 2016). Couples should also be informed about the positive interdependence of the symptoms and be aware that they change similarly within the dyad, in addition to the fact that during the postpartum period multiparous women are at greater risk of experiencing psychological difficulties.

In these programmes, partner support should be addressed because it has been identified as a protective factor for postpartum anxiety and depression disorders (Pilkington, Whelan, \& Milne, 2015). Even though several programmes including a partner component have been developed, and found to have significant benefits, they are still limited by a relatively low uptake and a tendency to exclude fathers. So, to promote adherence to these interventions, other than being delivered in health care centres, they could also be developed in a webbased format (Pilkington et al., 2015).

\section{Limitations and future directions}

The data were based on self-report measures, and there was evidence of selective attrition. This study had voluntary participation; those who agreed to participate may have been the ones exhibiting fewer anxiety and depression symptoms. The study had six assessment waves, over a time span of approximately 40 months. The fact that the fifth and sixth assessment waves had an interval of several months could be identified as a limitation, as there was a chance for several life events accounting for anxiety and depression symptoms to occur. Moreover, a high rate of dropouts existed in the last assessment wave. Piecewise dyadic growth curve models were estimated with the available data, considering the dropouts, and excluded missing data techniques, which can be pointed out as another limitation.

Future studies should include other measures, such as clinical diagnosis interviews, meant to compare data from self-report measures, and provide information on the prevalence of anxiety and depression disorders over pregnancy and the postpartum period. Research should continue to be developed using prospective longitudinal studies; however, the assessment waves should not be too far apart.

Finally, as significant variability was found in both men's and women's intercepts and slopes of anxiety and depression symptoms (Table 4), future research should consider additional variables to explain symptoms change over time such as the partner relationship quality, breastfeeding status, hormonal changes (for instance, oxytocin), or parent-child interaction. In the specific case of multiparous women, the factors contributing to the high risk for symptoms increase over the postpartum period need further exploration.

\section{Conclusions}

According to the present study's results, parents' anxiety and depression symptoms decline from early pregnancy to 3 months postpartum, and increase from 3 to 30 months 
postpartum, returning to the baseline levels. This study corroborates the existing literature considering that the transition to parenthood can last until the child's age of 2-3 years (Cowan et al., 1991). Reflecting the bio-psychosocial challenges faced by parents during the transition to parenthood, anxiety and depression symptoms trajectories over time were found to differ according to gender and parity, especially during the postpartum period. Primiparous women revealed low stable symptoms over the postpartum period, whereas multiparous women revealed the steepest symptoms increase (in comparison to primiparous men and women and multiparous men), pointing out a greater risk for psychological difficulties.

Additionally, anxiety and depression symptoms were positively correlated within-dyad over time. The findings of the present study have important implications for practice, namely for screening and intervention. Both prevention and intervention programmes aimed at reducing anxiety and depression symptoms over pregnancy and the postpartum period should include primiparous and multiparous men and women and pay particular attention to the period after 3 months postpartum.

\section{Note}

1. Model equations: $Y_{1 t i}=c_{1 i}+b_{1 i} T_{t i}+e 1_{t i^{\prime}} Y_{2 t i}=c_{2 i}+b_{2 i} T_{t i}+e_{2 t i .}$

\section{Acknowledgements}

We thank the couples that collaborated in this study. We also thank the researchers involved in data collection, and Iva Tendais and David Kenny who assisted in data analysis.

\section{Disclosure statement}

No potential conflict of interest was reported by the authors.

\section{Funding}

This work was provided by a doctoral grant from [FCT Fundação para a Ciência e a Tecnologia] [(SFRH/BD/50241/2009)] to the first author, and also by the FEDER Funds through the Programa Operacional Factores de Competitividade - COMPETE and by National Funds through FCT Fundação para a Ciência e a Tecnologia under the project PTDC/SAU/ SAP/116738/2010 (PI Bárbara Figueiredo).

\section{ORCID}

Catarina Canário (D) http://orcid.org/0000-0002-4206-2109

Bárbara Figueiredo (iD http://orcid.org/0000-0002-8209-7445

\section{References}

American Psychiatric Association. (2013). Diagnostic and statistical manual of mental disorders (5th ed.). Washington, DC: Author.

Areias, M., Kumar, R., Barros, H., \& Figueiredo, E. (1996). Correlates of postnatal depression in mothers and fathers. British Journal of Psychiatry, 169, 36-41. doi:10.1192/bjp.169.1.36 
Biaggio, A. M., Natalicio, L., \& Spielberger, C. D. (1976). The development and validation of an experimental Portuguese form of the State-Trait Anxiety Inventory. In C. D. Spielberger, \& R. DiasGuerrero (Eds.), Cross-cultural research on anxiety (pp. 29-40). Washington DC: Hemisphere/Wiley.

Breitkopf, C. R., Primeau, L., Levine, R. E., Olson, G. L., Wu, Z. H., \& Berenson, A. B. (2006). Anxiety symptoms during pregnancy and postpartum. Journal of Psychosomatic Obstetrics \& Gynecology, 27, 157-162. doi:10.1080/01674820500523521

Buist, A., Gotman, N., \& Yonkers, K. A. (2011). Generalized anxiety disorder: Course and risk factors in pregnancy. Journal of Affective Disorders, 131, 277-283. doi:10.1016/j.jad.2011.01.003

Canário, C., \& Figueiredo, B. (2016). Partner relationship from early pregnancy to 30 months postpartum: Gender and parity effects. Couple and Family Psychology: Research and Practice, 5, 226-239. doi:10.1037/cfp0000066

Chung, M. L., Moser, D. K., Lennie, T. A., \& Rayens, M. K. (2009). The effects of depressive symptoms and anxiety on quality of life in patients with heart failure and their spouses: Testing dyadic dynamics using Actor-Partner Interdependence Model. Journal of Psychosomatic Research, 67, 29-35. doi:10.1016/j.jpsychores.2009.01.009

Condon, J., \& Esuvaranathan, V. (1990). The influence of parity on the experience of pregnancy: A comparison of first- and second-time expectant couples. The British Journal of Medical Psychology, 63, 369-377. doi:10.1111/j.2044-8341.1990.tb01632.x

Cowan, P. A. (1991). Individual and family life transitions: A proposal for a new definition. In P.A. Cowan, \& M. Hetherington (Eds.), Family transitions (pp. 3-30). Hillsdale, NJ: Lawrence Erlbaum Associates.

Cowan, P. A., \& Cowan, C. P. (2012). Normative family transitions, couple relationship quality, and healthy child development. In F. Walsh (Ed.), Normal family processes: Growing diversity and complexity (pp. 428-451). New York, NY: Guilford Press.

Cowan, C. P., Cowan, P. A., Heming, G., \& Miller, N. B. (1991). Becoming a family: Marriage, parenting and child development. In P. A. Cowan, \& M. Hetherington (Eds.), Family transitions (pp. 79-110). Hillsdale, NJ: Lawrence Erlbaum Associates.

Cox, J. L., Holden, J. M., \& Sagovsky, R. (1987). Detection of postnatal depression: Development of the 10item Edinburgh postnatal depression scale. British Journal of Psychiatry, 150, 782-786. doi:10.1192/ jpb.150.6.782

Dekel, R., Vilchinsky, N., Liberman, G., Leibowitz, M., Khaskia, A., \& Mosseri, M. (2014). Marital satisfaction and depression among couples following men's acute coronary syndrome: Testing dyadic dynamics in a longitudinal design. British Journal of Health Psychology, 19, 347-362. doi:10.1111/bjhp.12042

Di Florio, A., Jones, L., Forty, L., Gordon-Smith, K., Robertson Blackmore, E., Heron, J., \& Craddock, I. (2014). Mood disorders and parity: A clue to the aetiology of the postpartum trigger. Journal of Affective Disorders, 152-154, 334-339. doi:10.1016/j.jad.2013.09.034

Dipietro, J., Costigan, K., \& Sipsma, H. (2008). Continuity in self-report measures of maternal anxiety, stress, and depressive symptoms from pregnancy through two years postpartum. Journal of Psychosomatic Obstetrics \& Gynecology, 29, 115-124. doi:10.1080/01674820701701546

Direção-Geral da Saúde. (2013). Registo do aleitamento materno: Relatório janeiro a dezembro de 2011. Stable url. Retrieved from. https://www.dgs.pt/?cr=24421

Don, B. P., \& Mickelson, K. D. (2012). Paternal postpartum depression: The role of maternal postpartum depression, spousal support, and relationship satisfaction. Couple and Family Psychology: Research and Practice, 1, 323-334. doi:10.1037/a0029148

Don, B. P., Chong, A., Biehle, S. N., \& Mickelson, K. D. (2014). Anxiety across the transition to parenthood: Change trajectories among low-risk parents. Anxiety, Stress, \& Coping: An International Journal, 27, 37-41. doi:10.1080/10615806.2014.903473

Dubber, S., Reck, C., Müller, M., \& Gawlik, S. (2015). Postpartum bonding:The role of perinatal depression, anxiety and maternal-fetal bonding during pregnancy. Archives of Women's Mental Health, 18, 187195. doi:10.1007/s00737-014-0445-4

Dudley, M., Roy, K., Kelk, N., \& Bernard, D. (2001). Psychological correlates of depression in fathers and mothers in the first postnatal year. Journal of Reproductive and Infant Psychology, 19, 187-202. doi:10.1080/02646830120073206

Duncan, T., Duncan, S., \& Strycker, L. (2006). An introduction to latent growth curve modeling: Concepts, issues and applications (2nd ed.). New Jersey: Lawrence Erlbaum Associates.

Escriba-Aguir, V., \& Artazcoz, L. (2011). Gender differences in postpartum depression: A longitudinal cohort study. Journal of Epidemiology \& Community Health, 65, 320-326. doi:10.1136/jech.2008.085894 
Escribe-Aguir, V., Gonzalez-Galarzo, M., Barona-Vilar, C., \& Artazcoz, L. (2008). Factors related to depression during pregnancy: Are there gender differences? Journal of Epidemiology \& Community Health, 62, 410-414. doi:10.1136/jech.2007.063016

Farr, S. L., Dietz, P. M., O'Hara, M. W., Burley, K., \& Ko, J. Y. (2014). Postpartum anxiety and comorbid depression in a population-based sample of women. Journal of Women's Health, 2, 120-128. doi:10.1089/jwh.2013.4438

Figueiredo, B., Pacheco, A., \& Costa, R. (2007). Depression during pregnancy and the postpartum period in adolescent and adult Portuguese mothers. Archives of Women's Mental Health, 10, 103-109. doi:10.1007/s00737-007-0178-8

Figueiredo, B., Teixeira, C., Conde, A., Pinto, A. R., \& Sarmento, P. (2009). Socio-demographic, medical and psychological characterization of the Júlio Dinis Maternity Hospital Outpatient Unit users. Revista Portuguesa de Psicologia, 41, 45-64.

Figueiredo, B., Canário, C., \& Field, T. (2014). Breastfeeding is negatively affected by prenatal depression and reduces postpartum depression. Psychological Medicine, 44, 927-936. doi:10.1017/ S0033291713001530

Garfield, C. F., Duncan, G., Rutsohn, J., McDade, T. W., Adam, E. K., Coley, R. L., \& Chase-Lansdale, P. L. (2014). A longitudinal study of paternal mental health during transition to fatherhood as young adults. Pediatrics, 133, 836-843. doi:10.1542/peds.2013-3262

Giallo, R., Woolhouse, H., Gartland, D., Hiscock, H., \& Brown, S. (2015). The emotional-behavioural functioning of children exposed to maternal depressive symptoms across pregnancy and early childhood: A prospective Australian pregnancy cohort study. European Child \& Adolescent Psychiatry, 24, 1233-1244. doi:10.1007/s00787-014-0672-2

Giardinelli, L., Innocenti, A., Benni, L., Stefanini, M., Lino, G., Lunardi, C., ... Faravelli, C. (2012). Depression and anxiety in perinatal period: Prevalence and risk factors in an Italian sample. Archives of Women's Mental Health, 15, 21-30. doi:10.1007/s00737-011-0249-8

Hakulinen, T., Laippala, P., \& Paunonen, M. (1998). Relationships between infant temperament, demographic variables, and family dynamics of childrearing families. Journal of Advanced Nursing, 27, 458-465. doi:10.1046/j.1365-2648.1998.00558.x

Henderson, J., \& Redshaw, M. (2016). Worries about labor and birth: A population-based study of outcomes for young primiparous women. Birth, 43, 151-158. doi:10.1111/birt.12219

Heron, J., O'Connor, T., Evans, J., Golding, J., \& Glover, V. (2004). The course of anxiety and depression through pregnancy and the postpartum in a community sample. Journal of Affective Disorders, 80 , 65-73. doi:10.1016/j.jad.2003.08.004

Huizink, A. C., Mulder, E. H., Robles de Medina, P. G., Visser, G. A., \& Buitelaar, J. K. (2004). Is pregnancy anxiety a distinctive syndrome? Early Human Development, 79, 81-91.

Keeton, C. P., Perry-Jenkins, M., \& Sayer, A. G. (2008). Sense of control predicts depressive and anxious symptoms across the transition to parenthood. Journal of Family Psychology, 22, 212-221. doi:10.1037/0893-3200.22.2.212

Kenny, D. A., Kashy, D. A., \& Cook, W. L. (2006). Dyadic data analysis. New York, NY:The Guilford Press.

Kerstis, B., Engström, G., Edlund, B., \& Aarts, C. (2013). Association between mothers'and fathers' depressive symptoms, sense of coherence and perception of their child's temperament in early parenthood in Sweden. Scandinavian Journal of Public Health, 41, 233-239. doi:10.1177/1403494812472006

Kessler, R. C., Keller, M. B., \& Wittchen, H. (2001). The epidemiology of generalized anxiety disorder. Psychiatric Clinics of North America, 24, 19-39. doi:10.1016/S0193-953X(05)70204-5

Kishi, R., McElmurry, B., Vonderheid, S., Altfeld, S., McFarlin, B., \& Tashiro, J. (2011). Japanese women's experiences from pregnancy through early postpartum period. Health Care for Women International, 32, 57-71. doi:10.1080/07399331003728634

Kjærgaard, H., Wijma, K., Dykes, A., \& Alehagen, S. (2008). Fear of childbirth in obstetrically low-risk nulliparous women in Sweden and Denmark. Journal of Reproductive \& Infant Psychology, 26, 340-350.

Lee, A. M., Lam, C. S. Y., Sze Mun Lau, H. W., Chong, S. K., \& Chui, D. Y.T. (2007). Prevalence, course, and risk factors for antenatal anxiety and depression. Obstetrics Gynecology, 110, 1102-1112.

Matthey, S., \& Ross-Hamid, C. (2012). Repeat testing on the Edinburgh Depression Scale and the HADS-A in pregnancy: Differentiating between transient and enduring distress. Journal of Affective Disorders, 141, 213-221. doi:10.1016/j.jad.2012.02.037

Matthey, S., Barnett, B., Ungerer, J., \& Waters, B. (2000). Paternal and maternal depressed mood during the transition to parenthood. Journal of Affective Disorders, 60, 75-85. doi:10.1016/\$01650327(99)00159-7 
Matthey, S., Valenti, B., Souter, K., \& Ross-Hamid, C. (2013). Comparison of four self-report measures and a generic mood question to screen for anxiety during pregnancy in English-speaking women. Journal of Affective Disorders, 148, 347-351. doi:10.1016/j.jad.2012.12.022

Melo, E. F., Cecatti, J. G., Pacagnella, R. C., Leite, D. F. B., Vulcani, D. E., \& Makuch, M. Y. (2012). The prevalence of perinatal depression and its associated factors in two different settings in Brazil. Journal of Affective Disorders, 136, 1204-1208. doi:10.1016/j.jad.2011.11.023

deMontigny, F., Girard, M., Lacharité, C., Dubeau, D., \& Devault, A. (2013). Psychosocial factors associated with paternal postnatal depression. Journal of Affective Disorders, 150, 44-49. doi:10.1016/j. jad.2013.01.048

Parfitt, Y., \& Ayers, S. (2009). The effect of post-natal symptoms of post-traumatic stress and depression on the couple's relationship and parent-baby bond. Journal of Reproductive and Infant Psychology, 27, 127-142. doi:10.1080/02646830802350831

Parfitt, Y., Pike, A., \& Ayers, S. (2013). The impact of parents' mental health on parent-baby interaction: A prospective study. Infant Behavior \& Development, 36, 599-608. doi:10.1016/j.infbeh.2013.06.003

Parker, M. L., Johnson, N., \& Ketring, S. A. (2012). Adult attachment and symptom distress : A dyadic analysis of couples in therapy. Journal of Family Therapy, 34, 321-344. doi:10.1111/j.14676427.2012.00598.x

Paulson, J., \& Bazemore, S. (2010). Prenatal and postpartum depression in fathers and its association with maternal depression: A meta-analysis. Journal of the American Medical Association, 303, 1961-1969. doi:10.1001/jama.2010.605

Perren, S., von Wyl, A., Bürgin, D., Simoni, H., \& von Klitzing, K. (2005). Depressive symptoms and psychosocial stress across the transition to parenthood: Associations with parental psychopathology and child difficulties. Journal of Psychosomatic Obstetrics \& Gynecology, 26, 173-183. doi:10.1080/01674820400028407

Pilkington, P. D., Whelan, T. A., \& Milne, L. C. (2015). A review of partner-inclusive interventions for preventing postnatal depression and anxiety. Clinical Psychologist, 19, 63-75. doi:10.1111/cp.12054

Pinquart, M., \& Teubert, D. (2010). A meta-analytic study of couple interventions during the transition to parenthood. Family Relations, 59, 221-231. doi:10.1111/j.1741-3729.2010.00597.x

Rholes, W., Simpson, J., Kohn, J., Wilson, C., Martin, A., Tran, S., \& Kashy, D. (2011). Attachment orientations and depression: A longitudinal study of new parents. Journal of Personality and Social Psychology, 100, 567-586. doi:10.1037/a0022802

Sekiyama, T., Nakatani, Y., Yu, X., Seki, Y., \& Sato-Suzuki, I. (2013). Increased blood serotonin concentrations are correlated with reduced tension/anxiety in healthy postpartum lactating women. Psychiatry Research, 209, 560-565. doi:10.1016/j.psychres.2013.03.009

Singley, D. B., \& Edwards, L. M. (2015). Men's perinatal mental health in the transition to fatherhood. Professional Psychology: Research and Practice, 46, 309-316. doi:10.1037/pro0000032

Skouteris, H., Wertheim, E. H., Rallis, S., Milgrom, J., \& Paxton, S. J. (2009). Depression and anxiety through pregnancy and the early postpartum: An examination of prospective relationships. Journal of Affective Disorders, 113, 303-308. doi:10.1016/j.jad.2008.06.002

Sockol, L. E., \& Battle, C. L. (2015). Maternal attitudes, depression, and anxiety in pregnant and postpartum multiparous women. Archives of Women's Mental Health, 18, 585-593. doi:10.1007/ s00737-015-0511-6

Spielberger, C. D., Gorsuch, R. L., Lushene, R., Vagg, P. R., \& Jacobs, G. A. (1983). Manual for the State-Trait Anxiety Inventory. Palo Alto, CA: Consulting Psychologists Press.

Stuebe, A. M., Grewen, K., \& Meltzer-Brody, S. (2013). Association between maternal mood and oxytocin response to breastfeeding. Journal of Women's Health, 22, 352-361. doi:10.1089/jwh.2012.3768

Sutter-Dallay, A., Cosnefroy, O., Glatigny-Dallay, E., Verdoux, H., \& Rascle, N. (2012). Evolution of perinatal depressive symptoms from pregnancy to two years postpartum in a low-risk sample:The MATQUID cohort. Journal of Affective Disorders, 139, 23-29. doi:10.1016/j.jad.2011.08.018

Tendais, I., Costa, R., Conde, A., \& Figueiredo, B. (2014). Screening for depression and anxiety disorders from pregnancy to postpartum with the EPDS and STAI. The Spanish Journal of Psychology, 17(e7), 1-9. doi:10.1017/sjp.2014.7 
van Bussel, J., Spitz, B., \& Demyttenaere, K. (2009). Anxiety in pregnant and postpartum women: An exploratory study of the role of maternal orientations. Journal of Affective Disorders, 114, 232-242. doi:10.1016/j.jad.2008.07.018

Wenzel, A. (2011). Anxiety symptoms during pregnancy and the postpartum period. In A. Wenzel, \& S. Stuart (Eds.), Anxiety in childbearing women: Diagnosis and treatment (pp. 21-35). Washington, DC: American Psychological Association. doi:10.1037/12302-001

Whisman, M. A., Davila, J., \& Goodman, S. H. (2011). Relationship adjustment, depression, and anxiety during pregnancy and the postpartum period. Journal of Family Psychology, 25, 375-383. doi:10.1037/ a0023790

Wynter, K., Rowe, H., \& Fisher, J. (2014). Interactions between perceptions of relationship quality and postnatal depressive symptoms in Australian, primiparous women and their partners. Australian Journal of Primary Health, 20, 174-181. doi:10.1071/PY12066

Zanardo, V., Gasparetto, S., Giustardi, A., \& Suppiej, F. (2009). Impact of anxiety in the puerperium on breast-feeding outcomes: Role of parity. Journal of Pediatric Gastroenterology Nutrition, 49, 631-634. doi:10.1097/MPG.0b013e31819e6446 